\title{
Is the Sodium an Activator of Immune System in Hypertension?
}

\author{
Patricio Araos and Cristián A Amador* \\ Laboratory of Renal Physiopathology, Institute of Biomedical Sciences, Universidad Autónoma de Chile, Chile
}

*Corresponding author: Cristián A Amador, Laboratory of Renal Physiopathology, Institute of Biomedical Sciences, Universidad Autónoma de Chile; Zip Code 8910060, Santiago, Chile

\begin{abstract}
ARTICLE INFO
Received: 幽 January 05, 2021

Published: 幽 January 12, 2021

Citation: Patricio Araos, Cristián A Amador. Is the Sodium an Activator of Immune System in Hypertension?. Biomed J Sci \& Tech Res 33(1)-2021. BJSTR. MS.ID.005355.

Abbreviations: APCs: Antigen Presenting Cells; AH: Arterial Hypertension; BP: Blood Pressure; DCs: Dendritic Cells; HS:

ABSTRACT

The sodium plays an essential role in human homeostasis and it has been described that a $30-50 \%$ of hypertensive patients are sensible to changes in their blood pressure after dietary high sodium intake. The last 20 years have been very active in trying to answer how the immune cells participate in hypertension, where the implications of increased sodium salt intake on the activation of the immune system have not been fully clarified. Even when some described mechanisms for macrophages, dendritic cells and lymphocytes proportion great insight for this understanding, the information concerning the different sodium compartments for its storage arise a new challenge for this area. This mini review highlights recent studies in different experimental settings regarding the effect of sodium as an activator for the immune system in hypertension and the mechanisms involved. We comment also the last studies suggesting the role of sodium as a regulator of immune cells in kidney medulla and extrarenal compartments that possibly may be involved in hypertension.
\end{abstract} High salt; IsoLG: Isolevuglandin; NADPH: Nicotinamide Adenine Dinucleotide Phosphate; PBMCs: Peripheral Blood Mononuclear Cells; SGK1: Serum Glucocorticoid Regulated Kinase 1
Keywords: Salt; Immune Cells; Sodium; Hypertension; Dendritic Cells; Macrophages; Lymphocytes; Kidney

\section{Introduction}

The sodium is essential for life in humans, where its balance is determined mainly by kidney function and by it consume that depends on social culture and people behavior. The World Health Organization has recommended adjusting the dietary sodium salt intake, since different studies suggest that high salt (HS) diet is strongly associated with cardiovascular diseases and blood pressure (BP) rise. The relationship between salt intake and BP has been well-established based on clinical and pre-clinical studies [1]. For instance, the International Study of Sodium, Potassium, and Blood Pressure study - INTERSALT - demonstrated in 10,079 patients that exist a direct association between sodium intake and BP increase [2]. More recently, interventional studies show that salt intake reduction decreases levels of BP in normotensive and hypertensive patients $[3,4]$, while a meta-analysis demonstrated that salt intake reduction in $2.5 \mathrm{~g} /$ day is associated with a $20 \%$ diminution in cardiovascular events [5]. The BP rise after HS intake has been classically related to the restricted renal capacity into excrete sodium, as consequence of the activation of the intrarenal renin-angiotensin aldosterone system and the reactive oxygen species formation [6-9]. In addition, HS intake may activate the sympathetic nervous system and to promote a direct vasoconstriction in blood vessels $[10,11]$, supporting a neurogenic mechanism.

However, and even when the activation of immune system has been associated to human arterial hypertension (AH) since 60s [12], the implications of increased sodium salt intake on the activation of the immune system have not been clarified. Here, we highlight the effects of sodium and how its distribution may participate on the immune system activation during $\mathrm{AH}$.

\section{Immune Cells in Hypertension}

The hypothesis for a causal role of immune cells in $\mathrm{AH}$ was proposed in 2007 starting with the Guzik's study [13]. In the last 20 years, different experimental approaches have been performed 
to evaluate how cells of the innate and adaptive immune systems participate in $\mathrm{AH}$, where monocytes-macrophages [14], dendritic cells (DCs) [15,16], and lymphocytes [17-19] present experimental evidence that supports their participation in $\mathrm{AH}$ [20]. In relation to the mechanisms involved, it has been described that monocytemacrophages act by increasing damage to target tissues [21], while DCs may produce isolevuglandin (IsoLG)-adduct, forming neoantigens after the activation of Nicotinamide adenine dinucleotide phosphate (NADPH) oxidase [22,23], promoting the activation of T-lymphocytes and the secretion of pro-inflammatory cytokines that favor to the sodium reabsorption and cardiovascular/ renal damage [24]. Additionally, although other components of the innate immune system, such as neutrophils and the complement system, have been less involved (and studied), their potential role in the development of $\mathrm{AH}$ cannot be ruled out $[25,26]$.

\section{Sodium Effect on Immune Cells in Hypertension}

Early studies demonstrated that hypertonic salt solutions are able to increase cytokine production from human and rabbit peripheral blood mononuclear cells (PBMCs) [27], supporting the idea that the microenvironment is relevant for the proinflammatory phenotype in $\mathrm{T}$ lymphocytes and monocytemacrophages [28]. Likewise, it was shown that the count of blood monocytes positively correlates with salt intake in normotensive patients [29]. Recently, Ruggeri et al. [30] demonstrated that CD14 ${ }^{+}$monocytes isolated from human PBMCs present an increase in IsoLG-adducts, and a greater secretion of pro-inflammatory cytokines, when cells are exposed to a HS concentration $(190 \mathrm{mM}$ of sodium chloride) [30]. Interestingly, this effect was prevented by inhibition of NADPH oxidase. In this study, the researchers observed that the transcriptome of monocytes exposed to a HS In Vitro, showed high expression of chemokine receptors and for proinflammatory cytokines, particularly interleukin-1 $\beta$ [30]. Together with other results, the authors suggest that a HS concentration promotes the activation of human monocytes mediated by NADPH oxidase and by the formation of IsoLG-adducts. In the same line, the authors demonstrated previously that the excess of dietary salt alters the gut microbiome leading to hypertension [31]. They found an increased accumulation of IsoLGs in the colon of hypertensive patients [31].

These findings were replicated in a murine model, where HS feeding also increased the IsoLG-adducts in mesenteric myeloid antigen-presenting cells (APCs), compared with animals that received normal salt diet [31]. The hypothesis proposes that sodium can enter to APCs via epithelial sodium channel (ENaC) leading to intracellular formation of IsoLGs, in a process regulated by the Serum and Glucocorticoid Kinase-1 (SGK1) and by NADPH oxidase [23,32]. This mechanism is quite interesting because suggest a new role for ENaC inhibition in the therapy for $\mathrm{AH}$ [33]. In addition, concerning to the role of $\mathrm{CD} 14^{+}$in APCs, it has been proposed that CD14 may participate with female sex hormone signaling modulating the pro-inflammatory activity favoring the salt-sensitive AH [34]. T lymphocytes favor the development of AH through the secretion of pro-inflammatory cytokines. In Vitro studies have shown that the increment of sodium salt concentration favors the T lymphocytes differentiation to T-helper (Th) ${ }_{17}$ cells in a SGK1-dependent way $[35,36]$. In this same line, knockout mice for Sgk1 in $\mathrm{CD}^{+}{ }^{+}$cells showed a prevention in $\mathrm{AH}$ and less vascular/ renal damage induced by angiotensin II and deoxycorticosteronesalt [37]. Interestingly, it has been observed that the expression of SGK1 is higher in $\mathrm{Th}_{17}$ and $\mathrm{T}_{\text {reg }}$ cells compared to other $\mathrm{T}$ lymphocytes [36], where the immunomodulatory capacity of Treg lymphocytes is inhibited by HS [38].

However, these evidence are not excluding the fact that sodium excess may activate other populations of Th lymphocytes [28], since the participation of $\mathrm{CD}^{+}$and $\mathrm{CD}^{+}$lymphocytes has been confirmed in different models of AH [20,39], indicating that SGK1 pathway could be a common signaling pathway within immune cells in salt-sensitive HA.

\section{Sodium Distribution and Its Possible Effects on Immune Response}

In the last 15 years, new studies have aimed the clinical impact of sodium distribution and storage in body compartments. One of the most discussed tissues is the skin; it has been demonstrated that sodium accumulation in skin interstitium is bounded to glycosaminoglycans [40]. This is quite interesting since monocytes-macrophages are proposed to be regulators of subcutaneous lymphatic system through the tonicity-responsive enhancer binding protein and the vascular endothelial growth factor-C [41]. Additionally, this mechanism is considered pivotal for monocytes-macrophages mobilization when skin present a HS content, representing a modulatory way for BP in patients. New experimental data is needed for this potential mechanism since high skin sodium content has been directly associated with cardiac hypertrophy in kidney disease [42]. The tunica intima is proposed also as a source of non-osmotic sodium storage and as a contributor to salt sensitivity [43]. These observations may proportionate new rationale for to explain the heterogeneity of patients in BP changes after HS intake, and how the tunica intima damage may alter the sodium balance in closely related diseases to $\mathrm{AH}$, such as renal disease and diabetes mellitus. However, new studies are necessary to determine how the endothelial cells in situation of HS may modulate the immune cells.

Finally, the kidney medulla it is well-recognized for represent a microenvironment with high osmolarity and sodium accumulation. The role of renal medulla on immunity is still controversial due to it hyperosmolarity has been reported to polarize APCs towards anti-inflammatory macrophages after renal transplant [44], suggesting that hypertonicity in renal medulla may modulate the pro-inflammatory status during kidney rejection or in the $\mathrm{AH}$. By contrast, a study by Berry et al. [45]. proposed that the hypersaline 
microenvironment set up a bactericidal activity in the kidney, by showing a high production of chemoattractant proteins from tubular epithelial cells [45].

\section{Conclusion}

In general, the available evidence suggest that increased local sodium levels can enhance the inflammatory potential of APCs, activating $\mathrm{T}$ lymphocytes, promoting inflammation and $\mathrm{AH}$. We need additional studies in order to describe the possible effects of sodium on innate immune cells, and whether the renal medulla, skin interstitium and the tunica intima microenvironment play a role in AH. However, and even when our knowledge is limited, the recommendation of sodium adjustment for patients in order to avoid cardiovascular diseases remains absolutely necessary.

\section{Acknowledgement}

We thank Pamela Torres for their administrative assistance, and the support of Fondecyt \#3201016 (P.A.) and Fondecyt \#1201251 (C.A.A.).

\section{Conflict of Interest}

\section{None.}

\section{References}

1. He FJ, MacGregor GA (2018) Role of salt intake in prevention of cardiovascular disease: Controversies and challenges. Nat Rev Cardiol 15(6): 371-377.

2. (1988) Intersalt: An international study of electrolyte excretion and blood pressure. Results for 24-hour urinary sodium and potassium excretion. Intersalt Cooperative Research Group. BMJ 297(6644): 319328.

3. Kotchen TA, Cowley AW, Frohlich ED (2013) Salt in health and disease--a delicate balance. N Engl J Med 368(13): 1229-1237.

4. Graudal NA, Hubeck Graudal T, Jurgens G (2020) Effects of low sodium diet versus high sodium diet on blood pressure, renin, aldosterone, catecholamines, cholesterol, and triglyceride. Cochrane Database Syst Rev 12: CD004022.

5. He FJ, Tan M, Ma Y, Mac Gregor GA (2020) Salt Reduction to Prevent Hypertension and Cardiovascular Disease: JACC State-of-the-Art Review. J Am Coll Cardiol 75(6): 632-647.

6. Granger JP, Schnackenberg CG (2000) Renal mechanisms of angiotensin II-induced hypertension. Semin Nephrol 20(5): 417-425.

7. Yamakoshi S, Ito O, Rong R, Ohsaki Y, Nakamura T, et al. (2020) High Salt Intake-Increased (Pro)renin Receptor Expression Is Exaggerated in the Kidney of Dahl Salt-Sensitive Rats. Hypertension 75(6): 1447-1454.

8. Touyz RM (2004) Reactive oxygen species, vascular oxidative stress, and redox signaling in hypertension: What is the clinical significance? Hypertension 44(3): 248-252.

9. Mattson DL, Dasinger JH, Abais Battad JM (2020) Amplification of SaltSensitive Hypertension and Kidney Damage by Immune Mechanisms. Am J Hypertens.

10. Fujita M, Fujita T (2013) The role of CNS in salt-sensitive hypertension. Curr Hypertens Rep 15(4): 390-394.

11. Batista MAC, Braga DCA, De Moura SAL, De Souza GHB, Dos Santos ODH, et al. (2020) Salt-dependent hypertension and inflammation: Targeting the gut-brain axis and the immune system with Brazilian green propolis. Inflammopharmacology 28(5): 1163-1182.
12. Koroskenyi K, Juba F, Vajda G (1961) Human vascular antigen complement consumption test of hypertensive patients (preliminary report). Experientia 17: 91-92.

13. Guzik TJ, Hoch NE, Brown KA, Mc Cann LA, Rahman A, et al. (2007) Role of the $\mathrm{T}$ cell in the genesis of angiotensin II induced hypertension and vascular dysfunction. J Exp Med 204(10): 2449-2460.

14. Wenzel P, Knorr M, Kossmann S, Stratmann J, Hausding M, et al. (2011) Lysozyme M-positive monocytes mediate angiotensin II-induced arterial hypertension and vascular dysfunction. Circulation 124(12): 1370-1381.

15. Hevia D, Araos P, Prado C, Fuentes Luppichini E, Rojas M, et al. (2018) Myeloid CD11 ${ }^{+}$Antigen-Presenting Cells Ablation Prevents Hypertension in Response to Angiotensin II Plus High-Salt Diet. Hypertension 71(4): 709-718.

16. Araos P, Prado C, Lozano M, Figueroa S, Espinoza A, et al. (2019) Dendritic cells are crucial for cardiovascular remodeling and modulate neutrophil gelatinase-associated lipocalin expression upon mineralocorticoid receptor activation. J Hypertens 37(7): 1482-1492.

17. Crowley SD, Song YS, Lin EE, Griffiths R, Kim HS, et al. (2010) Lymphocyte responses exacerbate angiotensin II-dependent hypertension. Am J Physiol Regul Integr Comp Physiol 298(4): R1089-1097.

18. Kasal DA, Barhoumi T, Li MW, Yamamoto N, Zdanovich E, et al. (2012) $\mathrm{T}$ regulatory lymphocytes prevent aldosterone-induced vascular injury. Hypertension 59(2): 324-330.

19. Trott DW, Thabet SR, Kirabo A, Saleh MA, Itani H, et al. (2014) Oligoclonal $\mathrm{CD}^{+} \mathrm{T}$ cells play a critical role in the development of hypertension. Hypertension 64(5): 1108-1115.

20. Drummond GR, Vinh A, Guzik TJ, Sobey CG (2019) Immune mechanisms of hypertension. Nat Rev Immunol 19(8): 517-532.

21. Justin Rucker A, Crowley SD (2017) The role of macrophages in hypertension and its complications. Pflugers Arch 469(3-4): 419-430.

22. Kirabo A, Fontana V, De Faria AP, Loperena R, Galindo CL, et al. (2014) DC isoketal-modified proteins activate $\mathrm{T}$ cells and promote hypertension. J Clin Invest 124(10): 4642-4656.

23. Barbaro NR, Foss JD, Kryshtal DO, Tsyba N, Kumaresan S, et al. (2017) Dendritic Cell Amiloride-Sensitive Channels Mediate Sodium-Induced Inflammation and Hypertension. Cell Rep 21(4): 1009-1020.

24. Norlander AE, Madhur MS (2017) Inflammatory cytokines regulate renal sodium transporters: How, where, and why? Am J Physiol Renal Physiol 313(2): F141-F144.

25. Araos P, Figueroa S, Amador CA (2020) The Role of Neutrophils in Hypertension. Int J Mol Sci 21(22): 8536.

26. Engstrom G, Hedblad B, Berglund G, Janzon L, Lindgarde F (2007) Plasma levels of complement $\mathrm{C}_{3}$ is associated with development of hypertension: A longitudinal cohort study. J Hum Hypertens 21(4): 276-282.

27. Junger WG, Coimbra R, Liu FC, Herdon Remelius C, Junger W, et al. (1997) Hypertonic saline resuscitation: A tool to modulate immune function in trauma patients? Shock 8(4): 235-241.

28. Wilck N, Balogh A, Marko L, Bartolomaeus H, Muller DN (2019) The role of sodium in modulating immune cell function. Nat Rev Nephrol 15(9): 546-558.

29. Yi B, Titze J, Rykova M, Feuerecker M, Vassilieva G, et al. (2015) Effects of dietary salt levels on monocytic cells and immune responses in healthy human subjects: a longitudinal study. Transl Res 166(1): 103-110.

30. Ruggeri Barbaro N, Van Beusecum J, Xiao L, Do Carmo L, Pitzer A, et al. (2020) Sodium activates human monocytes via the NADPH oxidase and isolevuglandin formation. Cardiovasc Res.

31. Ferguson JF, Aden LA, Barbaro NR, Van Beusecum JP, Xiao L, et al. (2019) High dietary salt-induced dendritic cell activation underlies microbial dysbiosis-associated hypertension. JCI Insight 5. 
32. Van Beusecum JP, Barbaro NR, Mc Dowell Z, Aden LA, Xiao L, et al. (2019) High Salt Activates CD11 $c^{+}$Antigen-presenting cells via SGK (Serum Glucocorticoid kinase) 1 to promote renal inflammation and SaltSensitive hypertension. Hypertension 74(3): 555-563.

33. Pitzer AL, Van Beusecum JP, Kleyman TR, Kirabo A (2020) ENaC in SaltSensitive Hypertension: Kidney and Beyond. Curr Hypertens Rep 22(9): 69.

34. Fehrenbach DJ, Abais Battad JM, Dasinger JH, Lund H, Keppel T, et al. (2021) Sexual Dimorphic Role of CD14 (Cluster of Differentiation 14) in Salt-Sensitive Hypertension and Renal Injury. Hypertension 77(1): 228240.

35. Kleinewietfeld M, Manzel A, Titze J, Kvakan H, Yosef N, et al. (2013) Sodium chloride drives autoimmune disease by the induction of pathogenic TH17 cells. Nature 496(7446): 518-522.

36. Wu C, Yosef N, Thalhamer T, Zhu C, Xiao S, et al. (2013) Induction of pathogenic TH17 cells by inducible salt-sensing kinase SGK1. Nature 496(7446): 513-517.

37. Norlander AE, Saleh MA, Pandey AK, Itani HA, Wu J, et al. (2017) A salt-sensing kinase in T lymphocytes, SGK1, drives hypertension and hypertensive end-organ damage. JCI Insight 2(13): e92801.

38. Hernandez AL, Kitz A, Wu C, Lowther DE, Rodriguez DM, et al. (2015) Sodium chloride inhibits the suppressive function of FOXP3 ${ }^{+}$regulatory T cells. J Clin Invest 125(11): 4212-4222.

\section{ISSN: 2574-1241}

DOI: $10.26717 /$ BJSTR.2021.33.005355

Cristián A Amador. Biomed J Sci \& Tech Res

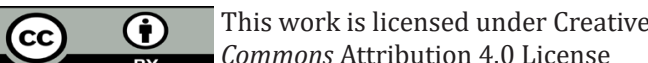

Submission Link: https://biomedres.us/submit-manuscript.php
39. Amador CA, Barrientos V, Peña J, Herrada AA, González M, et al. (2014) Spironolactone decreases DOCA-salt-induced organ damage by blocking the activation of $\mathrm{T}$ helper 17 and the downregulation of regulatory $\mathrm{T}$ lymphocytes. Hypertension 63(4): 797-803.

40. Titze J, Shakibaei M, Schafflhuber M, Schulze Tanzil G, Porst M, et al. (2004) Glycosaminoglycan polymerization may enable osmotically inactive $\mathrm{Na}^{+}$storage in the skin. Am J Physiol Heart Circ Physiol 287(1): H203-208.

41. Machnik A, Neuhofer W, Jantsch J, Dahlmann A, Tammela T, et al. (2009) Macrophages regulate salt-dependent volume and blood pressure by a vascular endothelial growth factor-C-dependent buffering mechanism. Nat Med 15(5): 545-552.

42. Schneider MP, Raff U, Kopp C, Scheppach JB, Toncar S, et al. (2017) Skin Sodium Concentration Correlates with Left Ventricular Hypertrophy in CKD. J Am Soc Nephrol 28(6): 1867-1876.

43. Olde Engberink RH, Rorije NM, Homan van der Heide JJ, van den Born BJ, Vogt L (2015) Role of the vascular wall in sodium homeostasis and salt sensitivity. J Am Soc Nephrol 26(4): 777-783.

44. Chessa F, Mathow D, Wang S, Hielscher T, Atzberger A, et al. (2016) The renal microenvironment modifies dendritic cell phenotype. Kidney Int 89(1): 82-94.

45. Berry MR, Mathews RJ, Ferdinand JR, Jing C, Loudon KW, et al. (2017) Renal Sodium Gradient Orchestrates a Dynamic Antibacterial Defense Zone. Cell 170(5): 860-874.

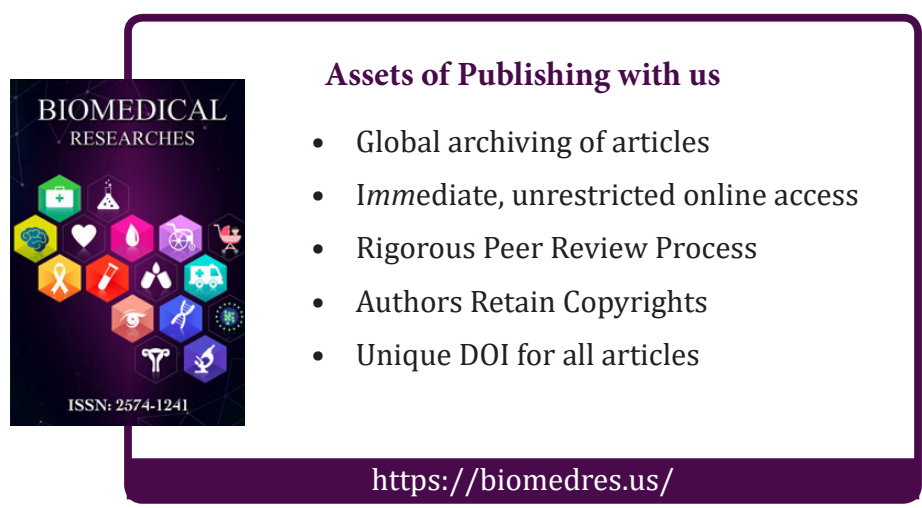

